\title{
Preclustering of Electrocardiographic Signals Using Left-to-Right Hidden Markov Models
}

\author{
Pau Micó ${ }^{1}$, David Cuesta ${ }^{1}$, and Daniel Novák ${ }^{2}$ \\ 1 Department of Systems Informatics and Computers,Polytechnic School of Alcoi, \\ Plaza Ferràndiz i Carbonell 2, 03801 Alcoi, Spain \\ \{pabmitor, dcuesta\}@disca.upv.es \\ 2 Department of Cybernetics, Czech Technical University in Prague, Czech Republic \\ xnovakd1@lab.felk.cvut.cz
}

\begin{abstract}
Holter signals are ambulatory long-term electrocardiographic (ECG) registers used to detect heart diseases which are difficult to find in normal ECGs. These signals normally include several channels and its duration is up to 48 hours. The principal problem for the cardiologists consists of the manual inspection of the whole holter ECG to find all those beats whose morphology differ from the normal synus rhythm. The later analisys of these arrhythmia beats yields a diagnostic from the pacient's heart condition. The Hidden Markov Models (HMM) can be used in ECG diagnosis avoiding the manual inspection. In this paper we improve the performance of the HMM clustering method introducing a preclustering stage in order to diminish the number of elements to be finally processed and reducing the global computational cost. An experimental comparative study is carried out, utilizing records form the MIT-BIH Arrhythmia database. Finally some results are presented in order to validate the procedure.
\end{abstract}

\section{Introduction}

HMMs [11], [3], [8] is a useful tool for biomedical signal analysis. The proposal would be to use them for clustering the beats contained in a Holter ECG signal in order to facilitate the cardiologist work. The problem lies within the great quantity of elements to be initially processed. Taking into account that the most of the beats will be grouped into the same cluster, it would be a good idea to reduce the number of redundant elements in a preclustering stage [2], [13]. After the preclustering, the more complex clustering algorithm (based on trained HMMs) will be applied.

In this paper we present a new method for HMMs quickly initialization (referred as left-to-right HMM). In addition, and improving the method proposed in [9], we are going to use left-to-right HMMs to drastically reduce in a preclustering stage the number of representative Holter beats. 


\section{Method}

Actually, the real goal of any Holter computer-aided process is to finally separate heart beats into different groups. The fact of classifying objects within these groups is known as clustering process. The accurate object feature extraction is needed in order to group these objects by means of dissimilitude evaluation. If the selected features does not represent the intrinsic quality of each object, the final results derived from clustering process will not become acceptable. We can approximate a biomedical signal using polygonal lines (segments) defined by two different features [6]: the amplitude of the sample and the duration of the segment. Through this beat approximation it is possible to initialize a left-to-right HMM to firstly cluster all the beats. In this case, the dissimilitude among objects is given by the HMM probability measure. Hence, the initialized model is applied to each beat and generates an output probability measure. This probability measure is used in order to calculate the dissimilitude estimator among beats. One beat will be clustered in certain group depending on its dissimilitude related to the cluster. Otherwise, if the dissimilitude is too high, beats should be unclassified. So, a general threshold is set and if the processed beat does not reach it, this beat will be directly labelled as unclassified. Threshold can be defined in two ways: fixing the probability or the dissimilitude level. Once all the beats have been (pre)clustered by the first model, the left-to-right HMM creation process will start again taking as the input a randomly selected unclassified beat. The loop will repeat until there are left no more unclassified beats. The whole process followed for HMM initialization and preclustering stages is shown in Figure 1. The input signal consists of a preprocessed ECG composed by a certain amount of segmented and normalized beats [2]. The algorithm output are the initialized HMMs and a lower number of beats to be processed in the later clustering stage. All these stages will be developed in the sections below.

\subsection{ECG Normalisation and Segmentation}

In most of the cases a 24 hour Holter is composed by more than 110, 000 beats [7]. The signal preprocessing plays a very important role for further analysis as classification or clustering. In this stage we solve the three main problems which normally arise in signal processing tasks: (i) characteristic point detection as a tool for signal preprocessing, (ii) baseline removal and (iii) signal denoising. As a result of the preprocessing, we will obtain (from the Holter) a clearly segmented set of beats. All the mentioned method has been developed under wavelet framework [9], [1] and [4].

\subsection{HMM Initialization}

A complete specification of an HMM [11] requires specification of two model parameters (the number of states, $N$; and the number of mixtures, $M$ ), and specification of the three probability measures (the state transition probability 


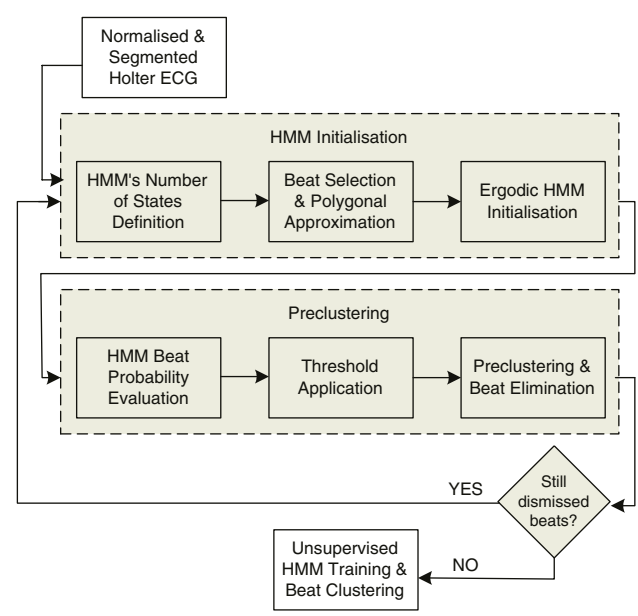

Fig. 1. Stages of the process.

distribution, $A$; the emission probability, $b$; and the initial state distribution, $\pi$ ). We will refer to all HMM parameters using the set:

$$
\lambda=\{A, B, \pi\}
$$

This type of statistical model has shown its capacities for certain types of biological signal modelling problem [6], [12] or [10]. In our case, to model an ECG throughout a left-to-right HMM (assuming that $M=1$ ), we will have to define the appropriate number of states in order to evaluate the three probability measures (Equation 1).

HMM's Number of States. The definition of the number of states of the model has serious implications in the way how the beats are approximated, and will be given by the morphology of the beat [6] that determines the minimum number of polygonal lines needed to approximate the beat. In this case, we just need 12 segments to approximate quite well the normal beat morphology [6] (Figure 2). This fact results in a left-to-right 12 - state HMM definition (Section 3).

Beat Selection and Polygonal Approximation. The HMM's number of states $N$, is closely related to the number of polygonal lines used for beat segmentation. In fact, the preclustering model briefly described in Section 2 is based on evaluating these polygonal lines passing through the model states (dissimilarity measure). The result of the polygonal approximation $\left(\theta_{k}\right)$ of the $k^{t h}$ beat from a sequence of segmented beats, consists of a set of points $\in \Re^{2}$ composed by two features: the duration $\left(t_{k i}\right)$, and the amplitude of the line segment $\left(h_{k i}\right)$. Once defined the number of states of the model $(N)$, we describe beat observation as follows: 


$$
\theta_{k}=\left[\left(t_{k 1}, h_{k 1}\right),\left(t_{k 2}, h_{k 2}\right), \ldots,\left(t_{k N}, h_{k N}\right)\right] \in \Re^{2}
$$

In this way, the question is how to keep every beat feature by just selecting the $N$ points $\in \Re^{2}$ in $E q$. 2 needed for the $N$-state HMM. To solve the problem we have used a simplified and faster version of the algorithm proposed in [6]. This new algorithm evaluates an error calculating the difference between the ordinate component from the point of the original record to its ordinate projection over the polygonal line used to approximate the interval processed, in order to quickly select the $N$ points.

Left-to-Right HMM Initialization. The left-to-right model assumes that each state is assigned to each polygonal line used for the ECG approximation. This topology reflects the sequential activity of the cardiac conduction system. Using the hidden Markov modelling approach, one observation (one of the lines from the beat polygonal approximation defined by the couple $\left.t_{k i}, h_{k i}\right)$ is generated by just one state transition, so there is a one-to-one registration between the observation sequence and the undelying state sequence. In order to initialize a left-to-right HMM we will choose randomly any beat (its 12-segment polygonal approximation) from the input ECG, resulting as follows:

- The state transition probability distribution A, will take the sub-diagonal form taking into account the left-to-right model definition.

- The initial state distribution $\pi$, will be centered on the initial state (giving the total likelihood to the state number one).

- The observation symbol probability distribution $b$, will depend on the selected beat's approximation. As this density estimation can be approximated with a Gaussian mixture (in this paper we assume that all the components have $d$-variate Gaussian distributions) [5], the mean value for each state will be set using the values of the 12 polygonal approximation selected points, that have been obtained from the concrete beat input observation sequence $\theta_{k}$ (Eq. 2). The variance for each state is calculated as a percentage of the mean (in our case, the 10\%).

On the other hand, the HMM lacks adaptibility if the inicialization is done as described above. In fact, if a concrete beat changes in some way (because baseline time shift, noise, etc.) but still belonging to a concrete morphology group, the HMM defined above will not be able to follow the variation and, consequently will classify the object into the wrong cluster. Using HMMs in this way help us to discard a big amount of similar (regular) beats although without ensuring that all of them with the same morphology are going to be clustered in the same group. This is the reason because preclustering models are only used to improve the unsupervised trained HMM stage and not for the clustering itself. A further development using unsupervised HMM training techniques (runned over the results from the preclustering stage) will provide larger HMMs (in terms of number of states) that will be able to adapt themselves to a concrete morphology without taking care of the different segment durations. 

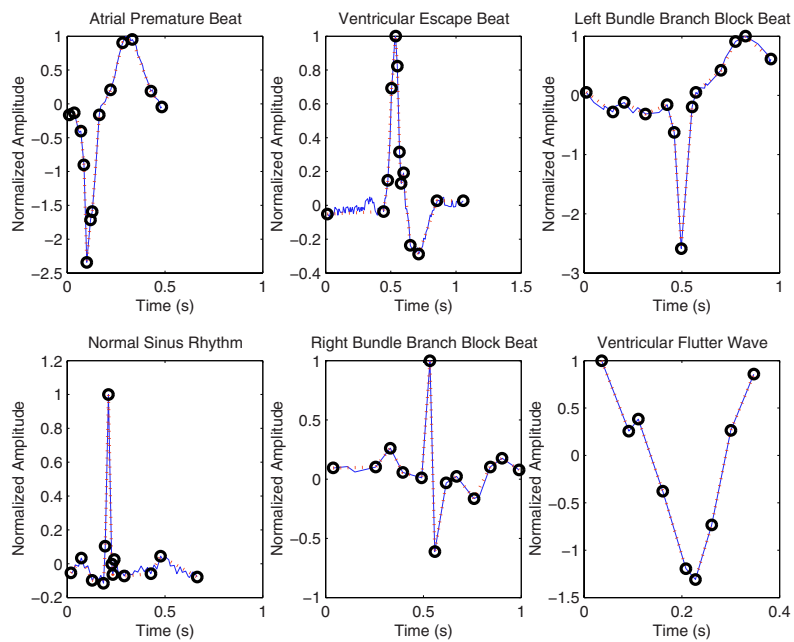

Fig. 2. 12-segment beat polygonal approximation determining a 12-state HMM. Different morphologies selected from initially unclassified beats are used to generate leftto-right HMMs to improve the preclustering stage.

\subsection{Preclustering}

Due to the great amount of beats contained in a Holter ECG, it is convenient to diminish (without loosing relevant information) the number of beats, in order to further alleviate computational burden.

The mathematical definition of clustering is as follows. Let $\mathcal{X}$ be our data set composed of vectors $x_{i}, \mathcal{X}=\left\{x_{1}, x_{2}, \ldots, x_{N}\right\}$. We define as an m-clustering of $\mathcal{X}$, the partition of $\mathcal{X}$ into $m$ sets (clusters), $\mathcal{C}_{1}, \ldots, \mathcal{C}_{m}$, so that the following three conditions are met:

$-\mathcal{C}_{i} \neq \emptyset, \quad i=1,2, \ldots, m$

$-\bigcup_{i=1}^{m} \mathcal{C}_{i}=\mathcal{X}$

$-\mathcal{C}_{i} \bigcap \mathcal{C}_{j}=\emptyset, \quad i \neq j, j=1, \ldots, m$

As we explain below, the developed preclustering algorithm is based on this definition and on the recurrent loop to identify and initializate the $m$ left-to-right HMMs for preclustering operations.

Beat Probability Evaluation. If we project the input beat $\theta_{k}$ to the model $h m m_{j}$ we obtain the probability that the beat is generated by the model, $\operatorname{Pr}\left(\theta_{k} \mid\right.$ $\left.h m m_{j}\right)$. Thus, when calculating this parameter for every beat from the input beat sequence of length $T$ and using every model obtained while repeating the loop from the preclustering model initialization stage (Figure 1), we will get the $P_{M \times T}$ beat probability matrix.The MIT-BIH Arrhythmia database [7] was used for results evaluation. 
Threshold Application. Depending on the beat probability matrix $P_{M \times T}$, it is possible to fix a threshold level in order to label (as non-classsified, we will use the symbol @) all those objects whose dissimilitude measure do not exceed the threshold. Thus, with an accurate threshold definition, we can easily simplify the preclustering stage from all those beats that have not been clearly evaluated by the models.

Preclustering and Beat Elimination. There are two parameters to apply at this stage: the threshold value and the dissimilitude distance. In the latter case we have used a very simple dissimilitude evaluation algorithm, since this distance is calculated for each beat as the maximum value from the corresponding column of the probability matrix, $P$. Thus, if the beat's dissimilitude exceeds the threshold, this beat will be clustered within the model that provides the maximum value (Eq. 3), otherwise it will be labelled as unclassified (@).

$$
c_{j} \mid \theta_{k}=\operatorname{argmax}_{1 \leq j \leq M}\left(P_{j, k}\right), \quad \forall \theta_{k} \in \Theta, \forall c_{i} \in C
$$

When all the beats have been (pre)clustered by the first model, the leftto-right HMM creation process will start again taking as the input a randomly selected unclassified beat. The loop will repeat until there are left no more unclassified beats. This process will generate so many left-to-right HMMs as necessary for the classification of the complete set of beats from the input ECG. Once every beat has been clustered, we have proceeded to the analisys of the cluster purity. The cluster purity is the percent of beats with the same morphology that have been grouped in that cluster. A high purity means that nearly every beat in the cluster has a similar morphology. So, and depending on the analysed cluster's purity we will be able to clean the cluster, deleting all those beats with a similar dissimilitude and keeping all those whose morphology maybe does not match exactly with the eliminated ones.

\section{Results}

Several input sets of segmented beats, from MIT-BIH database, have been prepared for the tests. These sets have been used both for HMMs evaluation and beat preclustering stage. A total of 18.700 beats have been processed in a series of ten different experiments, in each one of them threshold value has been changed. The results obtained from two of these test series are presented on Tables 1-2. Taking into account that every beat used in our tests has been previously labelled from MIT-BIH database, information and percents displayed within table cells follows the next format:

- The $1^{\text {st }}$ value from $c e l l_{i, j}$ gives us the number of beats labelled as $i$-morphology which have been classified to cluster $j$.

- The $2^{\text {nd }}$ value (among parenthesis, ()$)$ gives us the percentage of beats labelled as $i$-morphology in cluster $j$ from the total of beats that presents this concrete morphology. 
- The $3^{\text {rd }}$ value (bracketed,[]) gives us the percentage of beats labelled as $i$ morphology in cluster $j$ from the total of beats that have been classified in that cluster. We have referred to this parameter as cluster purity.

Beat labels used in tests have the following meaning:

$-\theta_{A}$ : atrial premature beat.

$-\theta_{E}$ : ventricular escape beat.

$-\theta_{L}$ : left bundle branch block beat.

$-\theta_{N}$ : normal sinus rhythm beat.

- $\theta_{R}$ : right bundle branch block beat.

- $\theta_{T}$ : ventricular flutter wave.

Table 1. Thresholded test to classify six different beat morphologies $\left(\theta_{i}\right)$ within seven possible clusters $\left(C_{j}\right)$, where cluster $C_{@}$ is used for threshold-dismissed beats.

\begin{tabular}{ccccccc}
\hline & $\theta_{A}$ & $\theta_{E}$ & $\theta_{L}$ & $\theta_{N}$ & $\theta_{R}$ & $\theta_{T}$ \\
\hline$C_{1}$ & $82(78.8)[97.6]$ & $0(0)[0]$ & $0(0)[0]$ & $2(0.5)[2.4]$ & $0(0)[0]$ & $0(0)[0]$ \\
$C_{2}$ & $0(0)[0]$ & $97(97)[52.7]$ & $0(0)[0]$ & $87(23.7)[47.2]$ & $0(0)[0]$ & $0(0)[0]$ \\
$C_{3}$ & $0(0)[0]$ & $0(0)[0]$ & $29(35.4)[16]$ & $74(20.2)[40.8]$ & $78(79.6)[43]$ & $0(0)[0]$ \\
$C_{4}$ & $0(0)[0]$ & $1(1)[0.5]$ & $0(0)[0]$ & $201(54.9)[99.5]$ & $0(0)[0]$ & $0(0)[0]$ \\
$C_{5}$ & $0(0)[0]$ & $0(0)[0]$ & $0(0)[0]$ & $2(0.7)[12.5]$ & $14(14.2)[87.5]$ & $0(0)[0]$ \\
$C_{6}$ & $0(0)[0]$ & $0(0)[0]$ & $0(0)[0]$ & $0(0)[0]$ & $0(0)[0]$ & $27(10.5)[100]$ \\
$C_{@}$ & $22(21.2)[7.2]$ & $2(2)[0.6]$ & $53(64.6)[16.8]$ & $0(0)[0]$ & $6(6.2)[1.9]$ & $231(89.5)[73.5]$ \\
\hline
\end{tabular}

As it is shown in Table 1 there are some unclassified beat in almost every morphology. Nevertheless, the purity percentage for clusters $C_{1}, C_{4}, C_{5}$ and $C_{6}$ is big enough to consider them as a good approximation in order to delete redundant beats and start the unsupervised HMM training stage. However it can be clearly observed how, ventricular escape beats (labelled as beats ${ }_{E}$ ) are the best grouped in the same cluster (97\%) in spite of its dirtiness in terms of purity $(52.7 \%)$ (Figure 3).

Table 2. Non-thresholded test to classify the ECG signal composed by two different beat morphologies $\left(\theta_{i}\right)$ within three possible clusters $\left(C_{j}\right)$.

\begin{tabular}{lcc}
\hline & $\theta_{R}$ & $\theta_{N}$ \\
\hline$C_{1}$ & $74(4)[77]$ & $22(2.4)[23]$ \\
$C_{2}$ & $70(3.8)[8]$ & {$[810][88.3][92]$} \\
$C_{3}$ & $1671(92)[95.2]$ & $85(9.2)[4.8]$ \\
\hline
\end{tabular}

In Table 2, there are only needed three from the eight HMM initially prepared for clustering the whole set of beats. In addition, the high purity percentage presented in clusters $C_{2}$ and $C_{3}$ reveals us that they fit almost perfectly with the preclustering models obtained from $\theta_{N}$ and $\theta_{R}$. Cluster $C_{1}$ has been used to group all those beats whose morphology have not been clearly identified (only the $4 \%$ of the total amount from $\theta_{R}$ and the $2.4 \%$ from $\theta_{N}$ ) (Figure 4 ). 


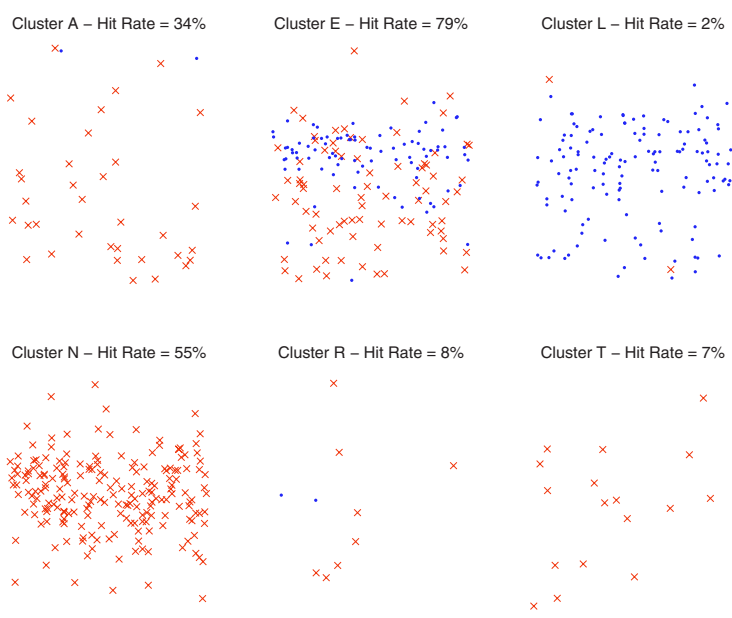

Fig. 3. Clusters obtained from results in Table 1. Rigth clustered beats are presented with vanes and badly ones with spots. Hit rate gives us the percent of beats classified within the right cluster.

Cluster 1 (96 beats)

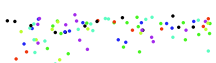

Cluster 4 ( 0 beats)

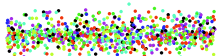

Cluster 2 ( 0 beats)

Cluster 3 ( 0 beats)

Cluster 5 ( 0 beats)

Cluster 6 ( 0 beats)

Fig. 4. Clusters obtained from results in Table 2. Consequently, it is possible to eliminate at least the $96.4 \%$ of the beats, what is a significant reduction of the input beat sequence size.

\section{Discussion and Conclusion}

We can conclude that it is very important to note that if more strict (in terms of probability) threshold is applied, the clusters are less contaminated. This is our final goal because of two reasons: first of all, to eliminate as much similar beats as possible in order to reduce the size of the input set of beats and alleviating, in this way, the computational burden; and secondly, to facilitate the HMM 
unsupervised training using this preclustering results. Although clustering with a very demanding threshold value is feasible, this fact presents the non-desirable effect of the non-classified objects increase, minimizing the preclustering stage performance. In spite of good results, it is very important to note the relevance of a correct model initialization, since the beat dissimilitude measure will depend on how the model fits with that beat. A badly initialized model will provide a low beat probability, and this fact will result in the labeling as non-classified or, what is worse, in the wrong clustering. Future work will be focused on improving each stage of the process by using dynamic programming techniques as Dynamic Time Warping (DTW).

\section{References}

1. Burrus, S.: Introduction to Wavelets and Wavelet Transforms. Prentice Hall, (1997)

2. Cuesta, D.: Estudio de métodos para procesamiento y agrupación de senales electrocardiográficas. Ph.D.Thesis, Valencia (2001)

3. Cuesta, D., Micó, P., and Novák, D.: Clustering electrocardiograph signals using Hidden Markov Models. European Medical and Biological Engineering Conference, Vienna (2002)

4. Daubechies, I.: Ten lectures on wavelets. (1992)

5. Duda, R., Hart, P. and Stork, D.: Pattern Classification. John Wiley \& Sons, (2001)

6. Koski, A.: Modelling ECG signals with hidden Markov models. Artificial Intelligence in Medicine, Vol. 8, (1996)

7. Mark,R., and Moody G.: MIT-BIH arrhythmia data base directory. Massachusetts Institute of Technology - Beth Israel Deaconess Medical Center, (1998)

8. Novák, D., Cuesta, D., Micó, P., and Lhotská, L.: Number of arrhythmia beats in Holter ECG: how many clusters? 25 ${ }^{\text {th }}$ Annual International Conference of the IEEE Engineering in Medicine and Biology Society, Cancún (2003)

9. Novák, D.: Electrocardiogram Signal Processing using Hidden Markov Models. Ph.D.Thesis, Prague (2004)

10. Obermaier, B.: Hidden Markov Models for online classification of single trial EEG data. Pattern Recognition Letters, Vol. 22,(2001)

11. Rabiner, R.: A Tutorial on Hidden Markov Models and Selected Applications in Speech Recognition. Proceedings of the IEEE, vol. 77, (1989)

12. Rezek, I. and Roberts, S.J.: Learning Ensemble Hidden Markov Models for Biosignal Analysis. 14th International Conference on Digital Signal Processing,(2002)

13. Vidal, E., and Marzal, A.: A Review and New Approaches for Automatic Segmentation of Speech Signals. Signal Processing V: Theories and Applications. Elsevier Science Publishers B. V, (1990) 